\title{
Maximal Inequalities for Dependent Random Variables and Applications
}

\author{
Soo Hak Sung \\ Department of Applied Mathematics, Pai Chai University, Taejon 302-735, South Korea
}

Correspondence should be addressed to Soo Hak Sung, sungsh@mail.pcu.ac.kr

Received 16 April 2008; Revised 3 June 2008; Accepted 7 July 2008

Recommended by Ondrej Dosly

For a sequence $\left\{X_{n}, n \geq 1\right\}$ of dependent square integrable random variables and a sequence $\left\{b_{n}, n \geq 1\right\}$ of positive numbers, we establish a maximal inequality for weighted sums of dependent random variables. Applying this inequality, we obtain the almost sure convergence of $\sum_{i=1}^{n} X_{i} / b_{i}$ and $\sum_{i=1}^{n} X_{i} / b_{n}$.

Copyright (C) 2008 Soo Hak Sung. This is an open access article distributed under the Creative Commons Attribution License, which permits unrestricted use, distribution, and reproduction in any medium, provided the original work is properly cited.

\section{Introduction}

Throughout this paper let $\left\{X_{n}, n \geq 1\right\}$ be a sequence of random variables defined on a probability space $(\Omega, \mathcal{F}, P)$ and let $\left\{b_{n}, n \geq 1\right\}$ be a sequence of positive numbers. We assume that there exists a sequence $\left\{\rho_{n}, n \geq 1\right\}$ of nonnegative constants such that

$$
\sup _{k \geq 1} E\left(X_{k} X_{k+n}\right) \leq \rho_{n}, \quad \text { for } n \geq 1 .
$$

In this paper, we establish a maximal inequality for weighted sums of the dependent random variables satisfying (1.1). Applying this inequality, we obtain under some suitable conditions on the sequence $\left\{\rho_{n}\right\}$ that

$$
\sum_{i=1}^{n} \frac{X_{i}}{b_{i}} \text { converges a.s. as } n \longrightarrow \infty
$$

and the strong law of large numbers (SLLN)

$$
\frac{\sum_{i=1}^{n} X_{i}}{b_{n}} \longrightarrow 0 \text { a.s. }
$$

Note that if $0<b_{n} \uparrow \infty$, then (1.2) implies (1.3) by the Kronecker lemma. 
For a sequence of dependent random variables satisfying (1.1), the SLLNs were established by Hu et al. [1,2] and Lyons [3]. Lyons [3] obtained an SLLN under the conditions that $\operatorname{Var}\left(X_{n}\right)=O(1)$ and $b_{n}=n$. Without condition $\operatorname{Var}\left(X_{n}\right)=O(1)$, Hu et al. [1] obtained an SLLN, where $b_{n}=n$. Hu et al. [2] also obtained an SLLN for more general sequence $\left\{b_{n}\right\}$ $\left(b_{n}=n\right.$ is replaced by $\left.n=O\left(b_{n}\right)\right)$.

For other results on the SLLN for a sequence of correlated random variables, see Chandra [4], Móricz [5, 6], and Serfling [7, 8].

In this paper, we give a sufficient condition under which (1.2) and (1.3) hold. Our results (partially) improve those of $\mathrm{Hu}$ et al. [1,2]. The technique used in our proof is the wellknown method of subsequences. Note that the maximal inequality is used in the method of subsequences. Our maximal inequality for weighted sums of the dependent random variables satisfying (1.1) is sharper than that of Hu et al. [2].

Throughout this paper, $\log x$ denotes the natural logarithm.

\section{Maximal inequalities for dependent random variables}

To prove the maximal inequality for weighted sums of dependent random variables satisfying (1.1), the following lemma is needed.

Lemma 2.1. Let $\left\{X_{n}, n \geq 1\right\}$ be a sequence of square integrable random variables satisfying (1.1). Let $\left\{b_{n}, n \geq 1\right\}$ be a sequence of positive numbers such that

$$
n \leq D b_{n} \quad \forall n \geq 1 \text { and some constant } D>0 .
$$

Then for all $n \geq 1, m>n$, and $\delta>0$,

$$
\sum_{i=n}^{m-1} \sum_{j=i+1}^{m} \frac{\left(E X_{i} X_{j}\right)^{+}}{b_{i} b_{j}} \leq \frac{D^{2} C_{\delta}}{\max \left\{(\log 2)^{\delta},(\log n)^{\delta}\right\}} \sum_{k=1}^{m-n} \frac{\rho_{k}}{k}(1+\log k)^{1+\delta},
$$

where $C_{\delta}=2^{\delta+1} \max \left\{1, \delta^{\delta} e^{-\delta}\right\}$.

Proof. For simplicity of notation, let $I_{n, m}=\sum_{i=n}^{m-1} \sum_{j=i+1}^{m}\left(E X_{i} X_{j}\right)^{+} /\left(b_{i} b_{j}\right)$. Then we get by (1.1) and (2.1) that for $1 \leq n<m$,

$$
\begin{aligned}
I_{n, m} & \leq \sum_{i=n}^{m-1} \sum_{j=i+1}^{m} \frac{\rho_{j-i}}{b_{i} b_{j}} \\
& \leq D^{2} \sum_{i=n}^{m-1} \sum_{j=i+1}^{m} \frac{\rho_{j-i}}{i j} \\
& =D^{2} \sum_{k=1}^{m-n} \sum_{i=n}^{m-k} \frac{\rho_{k}}{i(i+k)} \\
& =D^{2} \sum_{k=1}^{m-n} \frac{\rho_{k}}{k} \sum_{i=n}^{m-k}\left(\frac{1}{i}-\frac{1}{i+k}\right) \\
& \leq D^{2} \sum_{k=1}^{m-n} \frac{\rho_{k}}{k} \sum_{i=n}^{n+k-1} \frac{1}{i} .
\end{aligned}
$$


Soo Hak Sung

We next estimate $\sum_{i=n}^{n+k-1} 1 / i$. If $n=1$, then

$$
\sum_{i=n}^{n+k-1} \frac{1}{i}=\sum_{i=1}^{k} \frac{1}{i} \leq 1+\int_{1}^{k} \frac{1}{x} d x \leq 1+\log k \leq(1+\log k)^{1+\delta} .
$$

If $n \geq 2$, then

$$
\sum_{i=n}^{n+k-1} \frac{1}{i} \leq \int_{n-1}^{n+k-1} \frac{1}{x} d x=\log \left(1+\frac{k}{n-1}\right) \leq 2 \log \left(1+\frac{k}{n}\right) .
$$

The $\log (1+k / n)$ is estimated as follows:

$$
\begin{aligned}
& \log \left(1+\frac{k}{n}\right) \\
& \quad \leq \begin{cases}\log \left(1+\frac{1}{\sqrt{n}}\right) \leq \frac{(\log n)^{\delta}}{(\log n)^{\delta} \sqrt{n}} \leq \frac{(2 \delta)^{\delta} e^{-\delta}}{(\log n)^{\delta}} \leq(2 \delta)^{\delta} e^{-\delta} \frac{(1+\log k)^{1+\delta}}{(\log n)^{\delta}}, & \text { if } 1 \leq k \leq \sqrt{n}, \\
\log \left(1+\frac{k}{n}\right) \frac{(2 \log k)^{\delta}}{(\log n)^{\delta}} \leq 2^{\delta} \frac{(\log k)^{1+\delta}}{(\log n)^{\delta}} \leq 2^{\delta} \frac{(1+\log k)^{1+\delta}}{(\log n)^{\delta}}, & \text { if } k>\sqrt{n} .\end{cases}
\end{aligned}
$$

Thus, we have the desired estimate for $I_{n, m}$ :

$$
\begin{aligned}
I_{n, m} \leq \begin{cases}D^{2} \sum_{k=1}^{m-n} \frac{\rho_{k}}{k}(1+\log k)^{1+\delta}, & \text { if } n=1, \\
D^{2} \sum_{k=1}^{m-n} \frac{\rho_{k}}{k} \frac{2 \max \left\{2^{\delta},(2 \delta)^{\delta} e^{-\delta}\right\}}{(\log n)^{\delta}}(1+\log k)^{1+\delta}, & \text { if } n \geq 2,\end{cases} \\
\leq \frac{D^{2} 2^{\delta+1} \max \left\{1, \delta^{\delta} e^{-\delta}\right\}}{\max \left\{(\log 2)^{\delta},(\log n)^{\delta}\right\}} \sum_{k=1}^{m-n} \frac{\rho_{k}}{k}(1+\log k)^{1+\delta} .
\end{aligned}
$$

The following lemma is a maximal inequality for general dependent random variables.

Lemma 2.2. Let $\left\{X_{n}, n \geq 1\right\}$ be a sequence of square integrable random variables. Then for all $a \geq 0$ and $n \geq 1$,

$$
E\left(\max _{1 \leq k \leq n}\left|\sum_{i=a+1}^{a+k} X_{i}\right|^{2}\right) \leq\left(\frac{\log 2 n}{\log 2}\right)^{2}\left\{\sum_{i=a+1}^{a+n} E X_{i}^{2}+2 \sum_{i=a+1}^{a+n-1} \sum_{j=i+1}^{a+n}\left(E X_{i} X_{j}\right)^{+}\right\}
$$

Proof. Let $F_{a, n}$ be the joint distribution function of $X_{a+1}, \ldots, X_{a+n}$. Define a function $g$ on $\left\{F_{a, n}\right.$ : $a \geq 0, n \geq 1\}$ by

$$
g\left(F_{a, n}\right)=\sum_{i=a+1}^{a+n} E X_{i}^{2}+2 \sum_{i=a+1}^{a+n-1} \sum_{j=i+1}^{a+n}\left(E X_{i} X_{j}\right)^{+}
$$


Then we can easily obtain that for $a \geq 0, k \geq 1$, and $m \geq 1$,

$$
g\left(F_{a, k}\right)+g\left(F_{a+k, m}\right) \leq g\left(F_{a, k+m}\right) .
$$

Moreover, we have that for all $a \geq 0$ and $n \geq 1$,

$$
E\left(\sum_{i=a+1}^{a+n} X_{i}\right)^{2} \leq g\left(F_{a, n}\right)
$$

By Serfling's [9] generalization of the Rademacher-Menchoff maximal inequality for orthogonal random variables,

$$
E\left(\max _{1 \leq k \leq n}\left|\sum_{i=a+1}^{a+k} X_{i}\right|^{2}\right) \leq\left(\frac{\log 2 n}{\log 2}\right)^{2} g\left(F_{a, n}\right) .
$$

Thus, the result is proved.

Combining Lemmas 2.1 and 2.2 gives the following maximal inequality for weighted sums of dependent random variables satisfying (1.1).

Lemma 2.3. Let $\left\{X_{n}, n \geq 1\right\}$ be a sequence of square integrable random variables satisfying (1.1). Let $\left\{b_{n}, n \geq 1\right\}$ be a sequence of positive numbers satisfying (2.1). Then for all $n \geq 1, m>n$, and $\delta>0$,

$$
\begin{aligned}
& E\left(\max _{n \leq i \leq m}\left|\sum_{j=n}^{i} \frac{X_{j}}{b_{j}}\right|^{2}\right) \\
& \quad \leq\left(\frac{\log (2(m-n+1))}{\log 2}\right)^{2}\left\{\sum_{i=n}^{m} \frac{E X_{i}^{2}}{b_{i}^{2}}+\frac{2 D^{2} C_{\delta}}{\max \left\{(\log 2)^{\delta},(\log n)^{\delta}\right\}} \sum_{k=1}^{m-n} \frac{\rho_{k}}{k}(1+\log k)^{1+\delta}\right\},
\end{aligned}
$$

where $C_{\delta}=2^{\delta+1} \max \left\{1, \delta^{\delta} e^{-\delta}\right\}$.

\section{Almost surely convergent series and strong laws of large numbers}

In this section, we will assume that $\left\{X_{n}, n \geq 1\right\}$ is a sequence of square integrable random variables satisfying (1.1). A sufficient condition will be given under which (1.2) and (1.3) hold.

We first state and prove one of our main results. The proof is based on the well known method of subsequences. Our proof is similar to that of Hu et al. [2]. However, the maximal inequality (Lemma 2.3) used in the proof is sharper than that of $\mathrm{Hu}$ et al. [2].

Theorem 3.1. Let $\left\{X_{n}, n \geq 1\right\}$ be a sequence of square integrable random variables satisfying (1.1). Let $\left\{b_{n}, n \geq 1\right\}$ be a sequence of positive numbers satisfying (2.1). Suppose that the following conditions hold:

(i) $\sum_{n=1}^{\infty}(\log n)^{2} E X_{n}^{2} / b_{n}^{2}<\infty$,

(ii) $\sum_{n=1}^{\infty}(\log n)^{4+\delta} \rho_{n} / n<\infty$ for some $\delta>0$.

Then (1.2) holds. Furthermore, if $0<b_{n} \uparrow \infty$, then (1.3) holds. 
Proof. As noted in the introduction, if $0<b_{n} \uparrow \infty$, then (1.2) implies (1.3). To prove (1.2), let $S_{n}=\sum_{i=1}^{n} X_{i} / b_{i}$. By Lemma 2.1 with $\delta$ replaced by $3+\delta$, we have that for $m>n$,

$$
\begin{aligned}
E\left(S_{m}-S_{n}\right)^{2} & =\sum_{i=n+1}^{m} \frac{E X_{i}^{2}}{b_{i}^{2}}+2 \sum_{i=n+1}^{m-1} \sum_{j=i+1}^{m} \frac{E X_{i} X_{j}}{b_{i} b_{j}} \\
& \leq \sum_{i=n+1}^{m} \frac{E X_{i}^{2}}{b_{i}^{2}}+\frac{2 D^{2} C_{3+\delta}}{\max \left\{(\log 2)^{3+\delta},(\log n)^{3+\delta}\right\}} \sum_{k=1}^{m-n-1} \frac{\rho_{k}}{k}(1+\log k)^{4+\delta} \\
& \leq \sum_{i=n+1}^{\infty} \frac{E X_{i}^{2}}{b_{i}^{2}}+\frac{2 D^{2} C_{3+\delta}}{\max \left\{(\log 2)^{3+\delta},(\log n)^{3+\delta}\right\}} \sum_{k=1}^{\infty} \frac{\rho_{k}}{k}(1+\log k)^{4+\delta} \longrightarrow 0
\end{aligned}
$$

as $n \rightarrow \infty$ by (i) and (ii). Here $C_{3+\delta}=2^{\delta+4} \max \left\{1,(3+\delta)^{3+\delta} e^{-(3+\delta)}\right\}$. By the Cauchy convergence criterion, there exists a random variable $S$ such that $E\left(S_{n}-S\right)^{2} \rightarrow 0$ as $n \rightarrow \infty$. It is easy to see that $S_{2^{n}} \rightarrow S$ a.s. by the standard method. It remains to show that

$$
\max _{2^{n}<k \leq 2^{n+1}}\left|S_{k}-S_{2^{n}}\right| \longrightarrow 0 \text { a.s. } \quad \text { as } n \longrightarrow \infty .
$$

Using Lemma 2.3, (i), and (ii), we get that

$$
\begin{aligned}
\sum_{n=1}^{\infty} P & \left(\max _{2^{n}<k \leq 2^{n+1}}\left|S_{k}-S_{2^{n}}\right|>\epsilon\right) \\
& \leq \frac{1}{\epsilon^{2}} \sum_{n=1}^{\infty} E\left(\max _{2^{n}<k \leq 2^{n+1}}\left|S_{k}-S_{2^{n}}\right|^{2}\right) \\
& \leq \frac{1}{\epsilon^{2}} \sum_{n=1}^{\infty}\left(\frac{\log 2^{n+1}}{\log 2}\right)^{2}\left\{\sum_{i=2^{n}+1}^{2^{n+1}} \frac{E X_{i}^{2}}{b_{i}^{2}}+\frac{2 D^{2} C_{3+\delta}}{\left(\log \left(2^{n}+1\right)\right)^{3+\delta}} \sum_{k=1}^{2^{n}-1} \frac{\rho_{k}}{k}(1+\log k)^{4+\delta}\right\} \\
& \leq \frac{1}{\epsilon^{2}(\log 2)^{2}} \sum_{i=3}^{\infty} \frac{(\log (2 i))^{2} E X_{i}^{2}}{b_{i}^{2}}+\frac{2 D^{2} C_{3+\delta}}{\epsilon^{2}(\log 2)^{3+\delta}} \sum_{n=1}^{\infty} \frac{(n+1)^{2}}{n^{3+\delta}} \sum_{k=1}^{\infty} \frac{\rho_{k}}{k}(1+\log k)^{4+\delta}<\infty .
\end{aligned}
$$

Then (3.2) follows by the Borel-Cantelli lemma.

Remark 3.2. Hu et al. [2] proved Theorem 3.1 under (i) and (ii)'.

(ii) ${ }^{\prime} \sum_{n=1}^{\infty} \rho_{n} / n^{q}<\infty$ for some $0 \leq q<1$.

Since condition (ii) of Theorem 3.1 is weaker than (ii)', Theorem 3.1 improves the result of $\mathrm{Hu}$ et al. [2].

We can now establish the following SLLN if condition (2.1) on $\left\{b_{n}\right\}$ is replaced by the condition $0<b_{n} \uparrow \infty$.

Theorem 3.3. Let $\left\{X_{n}, n \geq 1\right\}$ be a sequence of square integrable random variables satisfying (1.1). Let $\left\{b_{n}, n \geq 1\right\}$ be a nondecreasing unbounded sequence of positive numbers. Suppose that the following conditions hold:

(i) $\sum_{n=1}^{\infty}(\log n)^{2} E X_{n}^{2} / b_{n}^{2}<\infty$, 
(ii) $\sum_{n=1}^{\infty} \rho_{n} \sum_{i=n+1}^{\infty}(\log i)^{2} / b_{i}^{2}<\infty$,

(iii) $\sum_{n=1}^{\infty} E X_{n}^{2} \sum_{i=n+1}^{\infty} \log i /\left(i b_{i}^{2}\right)<\infty$.

Then (1.3) holds.

To prove Theorem 3.3, we need the following lemma which is due to Fazekas and Klesov [10].

Lemma 3.4. Let $\left\{X_{n}, n \geq 1\right\}$ be a sequence of random variables and $\left\{b_{n}, n \geq 1\right\}$ be a nondecreasing unbounded sequence of positive numbers. Let $\left\{\alpha_{n}, n \geq 1\right\}$ be a sequence of nonnegative numbers. Assume that for each $n \geq 1$,

$$
E\left(\max _{1 \leq i \leq n}\left|\sum_{j=1}^{i} X_{j}\right|^{r}\right) \leq \sum_{i=1}^{n} \alpha_{i}, \quad \text { for some constant } r>0 .
$$

If $\sum_{n=1}^{\infty} \alpha_{n} / b_{n}^{r}<\infty$, then (1.3) holds.

Proof of Theorem 3.3. From Lemma 2.2,

$$
E\left(\max _{1 \leq i \leq n}\left|\sum_{j=1}^{i} X_{j}\right|^{2}\right) \leq\left(\frac{\log 2 n}{\log 2}\right)^{2}\left\{\sum_{i=1}^{n} E X_{i}^{2}+2 \sum_{i=1}^{n-1} \sum_{j=i+1}^{n}\left(E X_{i} X_{j}\right)^{+}\right\} .
$$

Define $\alpha_{n}=(\log 2 n / \log 2)^{2} A_{n}-(\log 2(n-1) / \log 2)^{2} A_{n-1}$ for $n \geq 1$, where $A_{0}=0$ and $A_{n}=$ $\sum_{i=1}^{n} E X_{i}^{2}+2 \sum_{i=1}^{n-1} \sum_{j=i+1}^{n}\left(E X_{i} X_{j}\right)^{+}$for $n \geq 1$. Then $E\left(\max _{1 \leq i \leq n}\left|\sum_{j=1}^{i} X_{j}\right|^{2}\right) \leq \sum_{i=1}^{n} \alpha_{i}$ and

$$
\begin{aligned}
\alpha_{n}= & \left(\frac{\log 2 n}{\log 2}\right)^{2}\left(A_{n}-A_{n-1}\right)+A_{n-1}\left\{\left(\frac{\log 2 n}{\log 2}\right)^{2}-\left(\frac{\log 2(n-1)}{\log 2}\right)^{2}\right\} \\
= & \left(\frac{\log 2 n}{\log 2}\right)^{2}\left\{E X_{n}^{2}+2 \sum_{i=1}^{n-1}\left(E X_{i} X_{n}\right)^{+}\right\} \\
& +\left\{\left(\frac{\log 2 n}{\log 2}\right)^{2}-\left(\frac{\log 2(n-1)}{\log 2}\right)^{2}\right\}\left\{\sum_{i=1}^{n-1} E X_{i}^{2}+2 \sum_{i=1}^{n-2} \sum_{j=i+1}^{n-1}\left(E X_{i} X_{j}\right)^{+}\right\} .
\end{aligned}
$$

By Lemma 3.4, it is enough to show that

$$
\begin{gathered}
\sum_{n=1}^{\infty} \frac{(\log 2 n)^{2} E X_{n}^{2}}{b_{n}^{2}}<\infty \\
\sum_{n=1}^{\infty} \frac{(\log 2 n)^{2}}{b_{n}^{2}} \sum_{i=1}^{n-1}\left(E X_{i} X_{n}\right)^{+}<\infty \\
\sum_{n=2}^{\infty} \frac{(\log 2 n)^{2}-(\log 2(n-1))^{2}}{b_{n}^{2}} \sum_{i=1}^{n-1} E X_{i}^{2}<\infty, \\
\sum_{n=3}^{\infty} \frac{(\log 2 n)^{2}-(\log 2(n-1))^{2}}{b_{n}^{2}} \sum_{i=1}^{n-2} \sum_{j=i+1}^{n-1}\left(E X_{i} X_{j}\right)^{+}<\infty .
\end{gathered}
$$

Clearly (3.7) holds by (i). It is easy to see that (3.8)-(3.10) hold, and the detailed proofs are omitted. 
The following corollary shows that condition (ii) of Theorem 3.3 can be simplified under the additional condition $(2.1)$ on $\left\{b_{n}\right\}$.

Corollary 3.5. Let $\left\{X_{n}, n \geq 1\right\}$ be a sequence of square integrable random variables satisfying (1.1). Let $\left\{b_{n}, n \geq 1\right\}$ be a nondecreasing unbounded sequence of positive numbers satisfying (2.1). Suppose that the following conditions hold:

(i) $\sum_{n=1}^{\infty}(\log n)^{2} E X_{n}^{2} / b_{n}^{2}<\infty$,

(ii) $\sum_{n=1}^{\infty}(\log n)^{2} \rho_{n} / n<\infty$,

(iii) $\sum_{n=1}^{\infty} E X_{n}^{2} \sum_{i=n+1}^{\infty} \log i /\left(i b_{i}^{2}\right)<\infty$.

Then (1.3) holds.

Proof. By (2.1), we have that

$$
\sum_{n=1}^{\infty} \rho_{n} \sum_{i=n+1}^{\infty} \frac{(\log i)^{2}}{b_{i}^{2}} \leq D^{2} \sum_{n=1}^{\infty} \rho_{n} \sum_{i=n+1}^{\infty} \frac{(\log i)^{2}}{i^{2}} \leq C \sum_{n=1}^{\infty} \rho_{n} \frac{(\log n)^{2}}{n}
$$

for some constant $C>0$. Thus the result follows by Theorem 3.3.

Remark 3.6. Condition (ii) of Corollary 3.5 is weaker than condition (ii) of Theorem 3.1. On the other hand, an additional condition is needed in Corollary 3.5 (namely condition (iii) above).

Using the following lemma, we can omit condition (iii) of Theorem 3.3 if conditions (2.1) and (3.12) on $\left\{b_{n}\right\}$ are satisfied. If $C_{1} n \leq b_{n} \leq C_{2} n^{\alpha}$ for all $n \geq 1$ and some constants $C_{1}>0, C_{2}>0$, and $\alpha>0$, then (2.1) and (3.12) hold.

Lemma 3.7. Let $\left\{b_{n}, n \geq 1\right\}$ be a nondecreasing unbounded sequence of positive numbers satisfying (2.1). If

$$
\limsup _{n \rightarrow \infty} \frac{\log b_{n}}{\log n}<\infty
$$

then

$$
\frac{b_{n}^{2}}{\log ^{2} n} \sum_{i=n}^{\infty} \frac{\log i}{i b_{i}^{2}}=O(1) .
$$

Proof. Without loss of generality, we may assume that $i \leq b_{i}$ for all $i \geq 1$.

Let fix $n$. For each $k \geq 1$, define $m_{k}$ by $m_{k}=\min \left\{i \geq n: b_{i} \geq k b_{n}\right\}$. Then $b_{m_{k}} \geq k b_{n}$ and $n=m_{1} \leq m_{2} \leq \cdots$. It follows that

$$
\begin{aligned}
\frac{b_{n}^{2}}{\log ^{2} n} \sum_{i=n}^{\infty} \frac{\log i}{i b_{i}^{2}} & =\frac{b_{n}^{2}}{\log ^{2} n} \sum_{k=1}^{\infty} \sum_{i=m_{k}}^{m_{k+1}-1} \frac{\log i}{i b_{i}^{2}} \leq \frac{b_{n}^{2}}{\log ^{2} n} \sum_{k=1}^{\infty} \frac{1}{b_{m_{k}}^{2}} \sum_{i=m_{k}}^{m_{k+1}-1} \frac{\log i}{i} \quad\left(\text { by } 0<b_{n} \uparrow\right) \\
& \leq \frac{b_{n}^{2}}{\log ^{2} n} \sum_{k=1}^{\infty} \frac{1}{\left(k b_{n}\right)^{2}} \sum_{i=m_{k}}^{m_{k+1}-1} \frac{\log i}{i} \\
& \leq \frac{1}{\log ^{2} n} \sum_{k=1}^{\infty} \frac{1}{k^{2}}\left(\sum_{i=1}^{3} \frac{\log i}{i}+\int_{3}^{m_{k+1}-1} \frac{\log x}{x} d x\right) \\
& \leq \frac{1}{\log ^{2} n} \sum_{k=1}^{\infty} \frac{1}{k^{2}}\left(\frac{\log 2}{2}+\frac{\log 3}{3}+\frac{\left(\log \left(m_{k+1}-1\right)\right)^{2}}{2}\right)
\end{aligned}
$$


where we assume in the case $m_{k+1}=m_{k}$, the sum $\sum_{i=m_{k}}^{m_{k+1}-1}=0$. Since $b_{i} \geq i$ for all $i \geq 1, b_{\left[k b_{n}\right]+1} \geq$ $\left[k b_{n}\right]+1 \geq k b_{n}$ and $m_{k} \leq\left[k b_{n}\right]+1 \leq k b_{n}+1$. So we have that

$$
\left(\log \left(m_{k+1}-1\right)\right)^{2} \leq\left(\log \left((k+1) b_{n}\right)\right)^{2} \leq 2\left\{(\log (k+1))^{2}+\left(\log b_{n}\right)^{2}\right\} .
$$

Substituting this into (3.14), (3.13) holds by (3.12).

The following example shows that Lemma 3.7 fails if (3.12) does not hold.

Example 3.8. Let $\phi(0)=1$ and $\phi(n)=2^{\phi(n-1)}$ for $n \geq 1$. Define a sequence $\left\{b_{n}, n \geq 1\right\}$ by $b_{n}=$ $\phi(k+1)$ if $\phi(k) \leq n<\phi(k+1)$. Then $0<b_{n} \uparrow \infty$ and $b_{n} \geq n$ for all $n \geq 1$. Since $b_{\phi(n)}=\phi(n+1)$, we obtain that

$$
\frac{\log b_{\phi(n)}}{\log \phi(n)}=\frac{\log \phi(n+1)}{\log \phi(n)}=\frac{\phi(n) \log 2}{\log \phi(n)} \longrightarrow \infty
$$

as $n \rightarrow \infty$. Hence (3.12) does not hold. We also obtain that for $n \geq 2$,

$$
\begin{aligned}
\frac{b_{\phi(n)}^{2}}{\log ^{2} \phi(n)} \sum_{i=\phi(n)}^{\infty} \frac{\log i}{i b_{i}^{2}} & \geq \frac{b_{\phi(n)}^{2}}{\log ^{2} \phi(n)} \sum_{i=\phi(n)}^{\phi(n+1)-1} \frac{\log i}{i b_{i}^{2}} \\
& =\frac{1}{\log ^{2} \phi(n)} \sum_{i=\phi(n)}^{\phi(n+1)-1} \frac{\log i}{i} \\
& \geq \frac{1}{\log ^{2} \phi(n)} \int_{\phi(n)}^{\phi(n+1)} \frac{\log x}{x} d x \\
& =\frac{1}{2}\left(\frac{\phi(n) \log 2}{\log \phi(n)}\right)^{2}-\frac{1}{2} \longrightarrow \infty
\end{aligned}
$$

as $n \rightarrow \infty$. So (3.13) does not hold.

If $\left\{b_{n}\right\}$ satisfies (2.1) and (3.12), then we can obtain the following SLLN.

Theorem 3.9. Let $\left\{X_{n}, n \geq 1\right\}$ be a sequence of square integrable random variables satisfying (1.1). Let $\left\{b_{n}, n \geq 1\right\}$ be a nondecreasing unbounded sequence of positive numbers satisfying (2.1) and (3.12). Suppose that the following conditions hold:

(i) $\sum_{n=1}^{\infty}(\log n)^{2} E X_{n}^{2} / b_{n}^{2}<\infty$.

(ii) $\sum_{n=1}^{\infty}(\log n)^{2} \rho_{n} / n<\infty$.

Then (1.3) holds.

Proof. By Lemma 3.7 and (i), we get

$$
\sum_{n=1}^{\infty} E X_{n}^{2} \sum_{i=n+1}^{\infty} \frac{\log i}{\left(i b_{i}^{2}\right)} \leq O(1) \sum_{n=1}^{\infty} \frac{\log ^{2}(n+1) E X_{n}^{2}}{b_{n+1}^{2}}<\infty,
$$

since $\log (n+1) / b_{n+1} \leq \log (n+1) / b_{n} \leq 2 \log n / b_{n}$ if $n \geq 2$. The result follows by Corollary 3.5. 
Remark 3.10. Under condition (3.12), Theorem 3.9 improves Theorem 3.1, since condition (ii) of Theorem 3.9 is weaker than condition (ii) of Theorem 3.1.

If $b_{n}=n$ for all $n \geq 1$, then $\left\{b_{n}\right\}$ satisfies (2.1) and (3.12). Hence we can obtain the following.

Corollary 3.11. Let $\left\{X_{n}, n \geq 1\right\}$ be a sequence of square integrable random variables satisfying (1.1). Suppose that the following conditions hold.

(i) $\sum_{n=1}^{\infty}(\log n)^{2} E X_{n}^{2} / n^{2}<\infty$.

(ii) $\sum_{n=1}^{\infty}(\log n)^{2} \rho_{n} / n<\infty$.

Then the SLLN holds. Namely,

$$
\frac{\sum_{i=1}^{n} X_{i}}{n} \longrightarrow 0 \text { a.s. }
$$

Remark 3.12. Lyons [3] proved an SLLN (3.19) under the conditions that $E X_{n}^{2}=O(1)$ and

$$
\sum_{n=1}^{\infty} \frac{\rho_{n}}{n}<\infty .
$$

When $E X_{n}^{2}=O(1)$, condition (i) of Corollary 3.11 is obviously satisfied. Hu et al. [1] proved an SLLN (3.19) under conditions (3.21) and (3.22):

$$
\begin{gathered}
\sum_{n=1}^{\infty} \frac{H\left(n^{\varphi+1}\right)}{n^{2}}<\infty, \\
\sum_{n=1}^{\infty} \frac{\rho_{n}}{n^{\varphi-1}}<\infty,
\end{gathered}
$$

where $\varphi=(1+\sqrt{5}) / 2(=1.618 \cdots)$ is the golden ratio, and $H(x)>0$ is a nondecreasing function on $(0, \infty)$ such that $E X_{n}^{2} \leq H(n)$ for all $n \geq 1$. Condition (ii) of Corollary 3.11 is weaker than (3.22). In general, condition (i) of Corollary 3.11 is not comparable with (3.21).

\section{Acknowledgments}

The author would like to thank the referees for helpful comments and suggestions that considerably improved the presentation of this paper. This work was supported by Korea Science and Engineering Foundation (KOSEF) grant funded by Korea government (MOST) (no. R01-2007-000-20053-0).

\section{References}

[1] T.-C. Hu, A. Rosalsky, and A. I. Volodin, "On the golden ratio, strong law, and first passage problem," The Mathematical Scientist, vol. 30, no. 2, pp. 77-86, 2005.

[2] T.-C. Hu, A. Rosalsky, and A. Volodin, "On convergence properties of sums of dependent random variables under second moment and covariance restrictions," Statistics \& Probability Letters. In press.

[3] R. Lyons, "Strong laws of large numbers for weakly correlated random variables," Michigan Mathematical Journal, vol. 35, no. 3, pp. 353-359, 1988. 
[4] T. K. Chandra, "Extensions of Rajchman's strong law of large numbers," Sankhyā. Series A, vol. 53, no. 1, pp. 118-121, 1991.

[5] F. Moricz, "The strong laws of large numbers for quasi-stationary sequences," Zeitschrift für Wahrscheinlichkeitstheorie und Verwandte Gebiete, vol. 38, no. 3, pp. 223-236, 1977.

[6] F. Móricz, "SLLN and convergence rates for nearly orthogonal sequences of random variables," Proceedings of the American Mathematical Society, vol. 95, no. 2, pp. 287-294, 1985.

[7] R. J. Serfling, "Convergence properties of $S_{n}$ under moment restrictions," The Annals of Mathematical Statistics, vol. 41, no. 4, pp. 1235-1248, 1970.

[8] R. J. Serfling, "On the strong law of large numbers and related results for quasistationary sequences," Theory of Probability and Its Applications, vol. 25, no. 1, pp. 187-191, 1980.

[9] R. J. Serfling, "Moment inequalities for the maximum cumulative sum," The Annals of Mathematical Statistics, vol. 41, no. 4, pp. 1227-1234, 1970.

[10] I. Fazekas and O. Klesov, "A general approach to the strong laws of large numbers," Theory of Probability and Its Applications, vol. 45, no. 3, pp. 436-449, 2001. 